\title{
BENEFÍCIOS DO TREINAMENTO FUNCIONAL PARA O EQUILÍBRIO E PROPRIOCEPÇÃO DE DEFICIENTES VISUAIS
}

\author{
BENEFITS OF FUNCTIONAL TRAINING FOR THE BALANCE AND PROPRIOCEPTION IN VISUALLY \\ IMPAIRED PERSONS
}

Artigo Original

Original ARTICLE

Artículo Original

\section{BENEFICIOS DEL ENTRENAMIENTO FUNCIONAL PARA EL EQUILIBRIO Y LA PROPIOCEPCIÓN DEDEFICIENTES VISUALES}

\section{Sara Teresinha Corazza' (Educadora Física) \\ Rafael Cunha Laux (Educador Físico) \\ Miriam Léa Strauss Foesch (Educadora Física) \\ Daniela Lopes dos Santos ${ }^{1}$ (Educadora Física) \\ Rafaella Righes Machado (Educadora Física) \\ Thuane Lopes Macedo \\ (Educadora Física) \\ Ana Carla Piovesan' (Fisioterapeuta) \\ Stela Paula Mezzomo ${ }^{1}$ \\ (Educadora Física)}

1. Universidade Federal de Santa Maria (UFSM), Santa Maria, RS, Brasil.

\section{Correspondência:}

Rafael Cunha Laux. Av. Roraima, 1000. Prédio 51, sala 1021. Campus Universitário km9. Bairro Camobi. Santa Maria, RS, Brasil. 97105-900. rafael-laux@hotmail.com

\section{RESUMO}

Introdução: A deficiência visual é uma limitação sensorial que afeta 6,5 milhões de pessoas somente no Brasil, chegando a 285 milhões no mundo. Os sujeitos afetados pela deficiência visual têm dificuldade maior para manter o equilíbrio, já que a visão é um dos principais sentidos envolvidos nesse complexo processo. Para melhorar essa capacidade físico-motora e, consequentemente, a qualidade de vida desses sujeitos, é necessário aperfeiçoar os outros sistemas de propriocepção. Um dos métodos para isso é o treinamento funcional. Objetivo: Analisar os efeitos do treinamento funcional sobre a propriocepção e o equilíbrio de pessoas com deficiência visual. Métodos: O presente estudo de caso analisou três sujeitos com cegueira total ou baixa visão, durante um programa de treinamento funcional que teve duração de 12 semanas, com duas sessões semanais. $O$ equilíbrio postural foi avaliado em uma plataforma de força, em condição bipodal e olhos vendados. A avaliação da propriocepção de membros superiores foi realizada com um cinesiômetro e a avaliação de membros inferiores foi realizada com a fixação de um flexímetro na articulação do joelho dominante do participante, ambas com os olhos vendados. Os resultados foram analisados de maneira descritiva e utilizando a técnica de análise de séries temporais. Resultados: Todos os sujeitos apresentaram melhora do equilíbrio postural. Com respeito à propriocepção do membro superior, o sujeito 1 (S1) apresentou melhora e quanto à propriocepção do membro inferior todos os sujeitos apresentaram melhora. Conclusão: Dessa forma, os resultados sugerem que o treinamento funcional influenciou positivamente o equilíbrio e a propriocepção desses indivíduos.

Descritores: pessoas com deficiência visual; qualidade de vida; equilíbrio postural; propriocepção.

\section{ABSTRACT}

Introduction: Visual impairment is a sensory disability that affects 6.5 million people in Brazil, reaching 285 million people worldwide. The subjects affected by visual impairment have more difficulty to maintain balance, as the view is one of the senses involved in this complex process. To improve such physical motor skills, and hence the life quality of these subjects, it is necessary to improve the other proprioception systems. One method for this is functional training. Objective: To analyze the effects of functional training on proprioception and balance of visually impaired persons. Methods: This case study evaluated three subjects with total blindness or low vision, during a functional training program that took 12 weeks with two sessions per week. Postural balance was evaluated on a force platform in bipedal condition and blindfolded. The evaluation of proprioception of the upper limbs was performed with a cinesiometer and the evaluation of the lower limbs was performed by fixing a fleximeter to the dominant kneejoint of the participant, both blindfolded. The results were analyzed descriptively using multivariate analysis of time series. Results: All subjects showed improvement in postural balance. With respect to the proprioception of upper limbs, the subject 1 (S1) showed improvement, and regarding the proprioception of the lower limbs all subjects showed improvement. Conclusion: Thus, the results suggest that functional training had a positive effect on balance and on proprioception of these individuals.

Keywords: visually impaired persons; quality of life; postural balance; proprioception.

\section{RESUMEN}

Introducción: La discapacidad visual es una deficiencia sensorial que afecta a 6,5 millones de personas en Brasil, llegando a 285 millones de personas en todo el mundo. Los sujetos afectados por daño visual tienen más dificultad para mantener el equilibrio, ya que la vista es uno de los sentidos que participan en este proceso complejo. Para mejorar estas habilidades físico motoras y, por lo tanto, la calidad de vida de estos sujetos, es necesario mejorar los otros sistemas de propiocepción. Un método para esto es el entrenamiento funcional. Objetivo: Analizar los efectos del entrenamiento funcional en la propiocepción yen el equilibrio de las personas con discapacidad visual. Métodos: Este estudio de caso analizó tres sujetos con ceguera total o baja visión durante un programa de entrenamiento funcional de 12 semanas, con dos sesiones por semana. El equilibrio postural fue evaluado sobre una plataforma de fuerza en la condición bípeda y con los ojos vendados. La evaluación de la propiocepción de las extremidades superiores fue realizada con un cinesiómetro y la evaluación de las extremidades inferiores fue realizada con la fijación de un flexímetro en la articulación de la rodilla dominante del participante, ambas con los ojos vendados. Los resultados 
fueron analizados de forma descriptiva y con el uso de análisis multivariado de series temporales. Resultados: Todos los sujetos mostraron una mejora en el equilibrio postural. Con respecto a la propiocepción del miembro superior, el sujeto 1 (S1) presentó una mejoría, y relativo al miembro inferior, todos los sujetos presentaron mejora. Conclusión: Por lo tanto, los resultados sugieren que el entrenamiento funcional tuvo un efecto positivo en el equilibrio postural y la propiocepción de estos individuos.

Descriptores: personas con daño visual; calidad de vida; balance postural; propiocepción.

\section{INTRODUÇÃO}

Atualmente, existem no mundo 285 milhões de pessoas com deficiência visual, dessas 39 milhões são cegas e as outras 246 milhões sofrem de perda de visão moderada ou severa'. Já no Brasil, são 6,5 milhões de pessoas com deficiência visual, sendo 582 mil cegas e seis milhões com baixa visão, conforme o censo de 2010 realizado pelo Instituto Brasileiro de Geografia e Estatística².

A deficiência visual (DV) é uma limitação sensorial que pode comprometer o desenvolvimento motor, afetivo, social e cognitivo do ser humano ${ }^{3}$. Em condições normais, os órgãos da visão contribuem com $85 \%$ dos estímulos encaminhados ao cérebro e são responsáveis pelo desenvolvimento e pela realização dos movimentos humanos. Portanto, a falta da visão implica em um comprometimento de atividades básicas que envolvem questões de segurança, integridade, autoimagem, orientação, percepção e aprendizagem ${ }^{4}$, prejudicando a interação social e possíveis oportunidades de estimulação motora, presentes na vida cotidiana ${ }^{5}$. Dessa forma, existem diferentes conceitos e valores que a sociedade impõe às pessoas com DV, gerando, muitas vezes, exclusão social. Uma forma de superar essa situação é proporcionar vivências sociais e motoras para que essa população alcance níveis de desenvolvimento dentro dos padrões considerados normais ${ }^{6-8}$.

Para promover o desenvolvimento motor desses sujeitos é necessário considerar que o controle das ações motoras depende do fluxo de sinais que estimulam os receptores sensoriais do corpo. Esses sinais são recebidos do ambiente externo pelos exteroceptores, como a visão, a audição e o tato, e internamente pelos proprioceptores, como os fusos musculares, os órgãos tendinosos de Golgi e o aparelho vestibular ${ }^{9}$. Então, para a execução motora são necessárias essas informações, que irão proporcionar condições para que o indivíduo se desloque no ambiente, mantendo sua noção de localização espacial, postural e posição de membros ${ }^{10,11}$.

O conjunto de informações fornecidas pela visão para a execução motora é denominado propriocepção visual ${ }^{12}$. Há casos em que o sujeito com DV utiliza as informações da propriocepção visual aprendidas em experiências anteriores ${ }^{9}$, e quando a ausência da visão se caracteriza por um longo e indefinido tempo, vai recrutar informações auditivas e proprioceptivas. Existe uma interação estreita entre os processos sensoriais e motores no controle postural, pois o movimento realizado necessariamente influencia as informações sensoriais fornecidas para o controle postural. Assim, a ação motora não dependerá somente das restrições mecânicas da tarefa, mas também da disponibilidade de informações sensoriais ${ }^{13}$.

Contudo, são necessários profissionais especializados para atuar com essa população, e entre eles destaca-se o professor de educação física, que através da ênfase na prática do exercício físico tem o papel de resgatar a funcionalidade e a independência dos DVs para a vida cotidiana ${ }^{14}$. Esse profissional deve utilizar técnicas e exercícios específicos que possibilitem ampliar a gama motora, a percepção corporal e espacial desses sujeitos ${ }^{15}$, mas também deve-se considerar que não são todos os exercícios que desenvolverão especificamente o equilíbrio, e se for esse o objetivo do treinamento, deverá ser escolhido um treinamento adequado e específico ${ }^{16}$.
Entre os exercícios físicos e métodos de treinamento propostos atualmente encontra-se o treinamento funcional, que utiliza padrão de movimentos, envolvendo todas as capacidades físicas através de movimentos multiarticulares e multiplanares. Seu objetivo é treinar funcionalidade através do princípio da transferência, onde se tenta reproduzir um gesto motor do cotidiano ou específico de alguma modalidade esportiva ${ }^{17}$.

Considerando o treinamento funcional como importante ferramenta para prevenção de lesões, aumento da autonomia, melhoria da saúde e capacidades físicas funcionais para os DV, surge o objetivo deste estudo que foi verificar os efeitos de um programa de treinamento funcional na propriocepção e no equilíbrio de pessoas com deficiência visual.

\section{MATERIAIS E MÉTODOS}

A pesquisa caracteriza-se como estudo de $\operatorname{caso}^{18}$. Todos os dados foram coletados no Laboratório de Biomecânica (LABIOMEC) do Centro de Educação Física e Desportos (CEFD), da Universidade Federal de Santa Maria (UFSM), Santa Maria, RS, Brasil. As avaliações foram realizadas após leitura e assinatura do Termo de Consentimento Livre e Esclarecido pelos participantes, conforme determina a Resolução 466/12 do Conselho Nacional de Saúde e o projeto aprovado no Comitê de Ética em pesquisa pelo CAAE no 31104314.2.0000.5346.

Os sujeitos do estudo foram selecionados intencionalmente na Associação de Deficientes Visuais (ACDV) da cidade de Santa Maria, RS. O critério de participação foi possuir deficiência visual (baixa visão ou cegueira), não possuir problemas de saúde como diabetes e hipertensão ${ }^{19}$, não usar medicamentos e concordar em participar da pesquisa.

Os sujeitos foram avaliados inicialmente com uma anamnese para selecionar os "casos" a serem estudados, conforme critérios anteriormente descritos. Após esse momento foram mensurados o equilíbrio e a propriocepção, aplicados à intervenção e reavaliados.

Na anamnese foram solicitados dados de identificação (sexo, idade, situação previdenciária, escolaridade); características da deficiência (baixa visão ou cegueira, congênita ou adquirida, tempo, causas e mobilidade); características sobre saúde (se é fumante, se usa bebida alcoólica, se possui algum comprometimento com a saúde, se utiliza medicamentos, a massa corporal, a estatura e o IMC), e seu braço e mão de domínio para realização do teste de propriocepção.

Avaliou-se o equilíbrio postural em condição bipodal, utilizando-se uma plataforma de força modelo OR6-6-2000 AMTI (Advanced Mechanical Technology Inc. 176 Waltham Street, Watertown, MA 02472-4800 EUA). Os sujeitos foram instruídos a permanecer o mais estático possível em cima da plataforma, na postura habitual e com os pés distanciados seguindo o alinhamento do quadril. Anteriormente, ao início da coleta, elaborou-se um molde do posicionamento dos pés de cada sujeito sob a plataforma para que mantivessem a mesma distância entre os pés na segunda coleta após as doze semanas de treinamento. Realizou-se três tentativas de olhos vendados para cada sujeito, com 30 segundos cada e com frequência de aquisição de $100 \mathrm{~Hz}$. Os dados de força foram utilizados no cálculo do centro de pressão (COP), e analisado a amplitude e velocidade de deslocamento 
nas direções anteroposterior (COPap) e médio-lateral (COPml), velocidade de deslocamento nas direções anteroposterior (COPvelap) e médio-lateral (COPvelml), conforme orientações de Cunha et al. ${ }^{20}$.

A avaliação da propriocepção de membros superiores foi realizada com o cinesiômetro (Santa Maria - RS - Brasil), conforme o protocolo de Paixão ${ }^{21}$. Para a coleta o sujeito sentou-se em frente ao instrumento, de olhos vendados, com seu braço de domínio sobre o braço móvel do cinesiômetro, estando esse em $0^{\circ}$. Durante a execução, o pesquisador moveu o braço do aparelho junto com o braço do sujeito, em cada um dos ângulos determinados: primeiramente $90^{\circ}$ para a direita em seguida $45^{\circ}$ para a esquerda e por último $105^{\circ}$ para a direita, retornando a posição inicial $0^{\circ}$. Após, foi solicitado ao sujeito que repetisse na mesma ordem, parando quando percebesse que eram as angulações corretas, conforme orientação anterior, para ser feito o registro. No final, a pontuação se deu por meio do cálculo do erro, resultante da média das três tentativas e das três posições.

Para verificar a propriocepção dos membros inferiores, foi utilizado um flexímetro (SANNY, São Bernardo do Campo, SP, Brasil). Este foi ajustado na articulação do joelho. Os indivíduos estavam com os olhos vendados. Foram mensurados os ângulos de $40^{\circ}$ e $20^{\circ}$, predeterminados por sorteio. Partindo-se de $90^{\circ}$ de flexão, a perna do avaliado foi movida passivamente em extensão até chegar à angulação (40\%/20\%) e o membro foi mantido por dez segundos nessa posição, retornando então à posição inicial. Após cinco segundos, o participante foi instruído a efetuar ativamente o mesmo movimento, parando assim que percebesse que a posição alvo tinha sido atingida ${ }^{22}$. Da mesma forma que a avaliação de propriocepção de membros superiores está apresentada ao a resultante da média de erro.

O experimento com treinamento funcional teve duração de 60 minutos, duas vezes por semana, durante 12 semanas, totalizando 24 aulas. Os exercícios desenvolvidos foram de sobrecarga corporal, de bases instáveis e de uso de equipamentos de ginástica para o aquecimento, conforme apresentado na Tabela 1. Precauções inerentes ao ensino da pessoa com deficiência visual foram observadas, como: iniciar atividades com diferentes possibilidades de assistência para que uma delas tenha sucesso; usar o mesmo tom de voz na abordagem individual; cuidar para não haver excesso de proteção; adaptar o ambiente, evitando a presença de muitos estímulos sonoros e com algum tipo de obstáculo; chamar cada aluno pelo nome, narrar atividades para que compreendam o que está acontecendo, incentivar participação e aprendizagem através de estímulos táteis, auditivos e visuais e solicitar sugestões de abordagem para melhorar o desempenho do aluno ${ }^{23}$.

As aulas foram organizadas em forma de circuito, intercalando exercícios aeróbicos com exercícios funcionais, seguindo um nível de dificuldade progressivo desde a primeira até a última aula. Ex: Na primeira aula, os exercícios funcionais eram de baixa complexidade (bíceps/ tríceps com gymstick), Nas últimas aulas foram ministrados exercícios de alta complexidade como burpee e prancha ventral. A intervenção foi realizada em uma sala multiuso preparada em estações para o treinamento em forma de circuito funcional.

\section{Análise estatística}

Os dados foram analisados de maneira descritiva (médias, frequências relativas e absolutas), e apresentados usando a técnica de análise de séries temporais ${ }^{18}$.

\section{RESULTADOS}

Os sujeitos estudados são do sexo masculino e serão apresentados como S seguido de número (Ex: S1, S2 e S3), conforme Tabela 2.

O tipo de deficiência de $S 1$ é cegueira total, adquirida por glaucoma e deslocamento de retina, usa bengala e é independente para todas as atividades. O S2 tem baixa visão, adquirida por glaucoma e se locomove sozinho. O S3 também tem deficiência do tipo baixa visão,
Tabela 1. Estrutura da intervenção com treinamento funcional.

\begin{tabular}{c|c|c}
\hline Aulas & Tempo (min) & \multicolumn{1}{c}{ Descrição atividades realizadas } \\
\hline Aquecimento & 10 & $\begin{array}{l}\text { Atividades no slide, esteira, bicicleta, elíptico, } \\
\text { corrida estacionária e/ou corrida lateral. }\end{array}$ \\
\hline Treinamento & \multirow{2}{*}{$\begin{array}{l}\text { Agachamento simples no suspenso, remada } \\
\text { fechada no suspenso, prancha ventral, tríceps na } \\
\text { estação de musculação, bíceps com um pé no disco } \\
\text { de equilíbrio, agachamento com bola nas costas, } \\
\text { sobe e desce no step, panturrilha com medicine ball, } \\
\text { corrida estacionária no jump, flexão de braços no } \\
\text { suporte, tríceps no gymstick, corrida com cinto de } \\
\text { tração, bíceps no disco de equilíbrio, abdominais } \\
\text { na bola suiça, afundo com um pé no step, corrida } \\
\text { no suspenso, afundo no suspenso, bíceps com um } \\
\text { pé no bosu, burpee, afundo com elevação de braços, } \\
\text { bíceps sentado na bola, agachamento nojump, sobe } \\
\text { e desce do step pela frente, tríceps com gymstick, } \\
\text { trote-4 passos para frente/4 passos para trás, tríceps } \\
\text { no suspenso com os joelhos no chão, equilíbrio no } \\
\text { bosu, panturrilha e tríceps com elástico, equilíbrio } \\
\text { com um pé em cada disco, corrida estacionária no } \\
\text { suspenso, remada aberta no suspenso, abdominal } \\
\text { com os pés na bola. }\end{array}$} \\
\hline Alongamento & $\begin{array}{l}\text { Alongamento no solo, alongamento na barra, } \\
\text { alongamento em pé. }\end{array}$ \\
\hline
\end{tabular}

adquirida por retinose pigmentar e se locomove com o auxílio de pessoa vidente, porém é independente para as atividades cotidianas como autocuidado, incluindo tarefas de escovar os dentes, pentear os cabelos, vestir-se, tomar banho, calçar sapatos, alimentar-se, beber água, fazer uso do vaso sanitário; e tarefas cotidianas como lavar louça, arrumar a cama, varrer a casa e usar o telefone (Tabela 2).

Na Tabela 3, dados sobre equilíbrio postural. Variáveis analisadas pré e pós teste: amplitude de deslocamento anteroposterior (COPap) e médio-lateral (COPml) e velocidade de deslocamento anteroposterior (COPvelap) e médio-lateral (COPvelml). Houve melhora em todas variáveis para todos os sujeitos no pós-teste.

Na Tabela 4, são apresentadas as médias de erro do pré e do pós teste da propriocepção de membros superiores e inferiores. O sujeito S2 apresentou melhora em ambos os testes. Já os sujeitos S1 e S3 apresentaram melhoras somente no teste de membros inferiores.

\section{DISCUSSÃO}

Após a intervenção, observou-se melhora nos resultados dos três sujeitos nas variáveis do equilíbrio, o que vem ao encontro do estudo de Silva et al. ${ }^{24}$, que apontou que a dança proporcionou ganhos tanto no equilíbrio estático quanto no dinâmico das pessoas com deficiência visual do seu estudo, na faixa etária de 12- 60 anos, de ambos os sexos. Souza et al. ${ }^{25}$ verificaram o efeito da ginástica artística para essa mesma população e concluíram que no final de um programa de dezoito aulas com os fundamentos da ginástica artística houve uma melhora no equilíbrio.

No estudo de caso de Soares et al. ${ }^{14}$ que utilizaram o método de observação para verificar a melhora do equilíbrio entre outras variáveis

Tabela 2. Caracterização dos sujeitos.

\begin{tabular}{c|c|c|c}
\hline & S1 & S2 & S3 \\
\hline Idade (anos) & 20 & 39 & 41 \\
\hline Massa Corporal (kg) & 60,3 & 61,1 & 80,5 \\
\hline Estatura (cm) & 184 & 168 & 181 \\
\hline Tipo de deficiência & CT & BV & BV \\
\hline $\begin{array}{c}\text { Tempo com a deficiência } \\
\text { (anos) }\end{array}$ & 12 & 7 & 18
\end{tabular}

CT: Cegueira Total; BV: Baixa Visão. 
Tabela 3, Dados sobre equilíbrio postural,

\begin{tabular}{c|c|c|c|c|c|c}
\hline \multirow{2}{*}{$\begin{array}{c}\text { Váriaveis do } \\
\text { equilibrio }\end{array}$} & \multicolumn{2}{|c|}{ S1 } & \multicolumn{2}{c|}{ S2 } & \multicolumn{2}{c}{ S3 } \\
\cline { 2 - 7 } & Pré & Pós & Pré & Pós & Pré & Pós \\
\hline COPap & 2,47 & 1,89 & 2,31 & 1,61 & 1,88 & 1,00 \\
\hline COPml & 2,86 & 1,12 & 2,61 & 1,38 & 1,43 & 0,88 \\
\hline COPvelap & 1,91 & 0,88 & 3,12 & 1,51 & 1,60 & 1,22 \\
\hline COPvelml & 1,34 & 0,65 & 2,48 & 2,02 & 1,41 & 0,72 \\
\hline
\end{tabular}

COPap: amplitude de deslocamento anteroposterior $(\mathrm{cm})$; COPml: amplitude de deslocamento médio-lateral $(\mathrm{cm})$; COPvelap: velocidade de deslocamento anteroposterior $(\mathrm{cm} / \mathrm{s})$; COPvelml: velocidade de deslocamento médio-lateral $(\mathrm{cm} / \mathrm{s})$,

Tabela 4, Média de erro da propriocepção de MS e MI

\begin{tabular}{c|c|c|c|c|c|c}
\hline \multirow{2}{*}{ Propriocepção } & \multicolumn{2}{|c|}{ S1 } & \multicolumn{2}{c|}{ S2 } & \multicolumn{2}{c}{ S3 } \\
\cline { 2 - 7 } & Pré & Pós & Pré & Pós & Pré & Pós \\
\hline Membro Superior & $8,3^{\circ}$ & $8,3^{\circ}$ & $10,0^{\circ}$ & $1,6^{\circ}$ & $7,3^{\circ}$ & $8,3^{\circ}$ \\
\hline Membro Inferior & $3,5^{\circ}$ & $2,0^{\circ}$ & $6,5^{\circ}$ & $3,5^{\circ}$ & $5,0^{\circ}$ & $2,5^{\circ}$ \\
\hline
\end{tabular}

Resultados apresentados em média do erro em graus,

motoras, percebeu-se uma melhora no equilíbrio conforme avançava o processo de intervenção, corroborando com resultados desse estudo.

Silva et al. ${ }^{26}$ em seu estudo com crianças e adolescentes asmáticos e não-asmáticos reafirmaram a importância da visão para o equilíbrio ao realizar testes na plataforma de força, com olhos abertos e fechados, salientando que o teste com olhos fechados dificultou a representação adequada do corpo dos indivíduos, no ambiente do teste. Isto nos aponta a importância de enfatizar no treinamento funcional a prática da propriocepção em indivíduos com deficiência visual, pois os sistemas sensoriais identificarão outros elementos importantes para o indivíduo conseguir controlar seu corpo no espaço.

No estudo de Antes et al. ${ }^{27}$ que analisou a estabilidade postural e a propriocepção de idosas fisicamente ativas, foi encontrada correlação positiva entre a propriocepção e a idade e entre a propriocepção e a estabilidade postural, o que vem ao encontro do presente estudo, enfatizando a importância da propriocepção para o equilíbrio.

Na propriocepção, os resultados foram positivos para todos os sujeitos nos testes de membros inferiores. O sujeito $\$ 2$ obteve resultados positivos tanto para os membros superiores quanto para os inferiores, e somando aos resultados positivos do equilíbrio, podemos associar esses resultados aos efeitos do treinamento funcional, somados a sua independência na locomoção (sem bengala ou pessoa vidente), e ao menor tempo de deficiência (baixa visão) ${ }^{28}$.

Interpretou-se o bom desempenho do $\$ 2$ em todas as variáveis nos pós testes, através do estudo de Soares et al..$^{14}$ que afirmam ser factível a compreensão de que déficits motores não sejam inerentes à condição de DV, mas a falta de estimulação que ocorre decorrente da falta de profissionais especializados para atender esse público ou o medo que a própria família tem do DV se machucar durante a prática dos exercícios físicos. Entretanto, desenvolver atividades para estimulação psicomotora é de extrema necessidade.

Antes et al. ${ }^{29}$ em seu estudo sobre propriocepção de joelho em jovens e idosas praticantes de atividades físicas, observaram pequena diferença na média de erro entre idosas e jovens, devido ao fato que ambos grupos eram fisicamente ativos. No entanto, quando se compara as médias do grupo de jovens com os sujeitos deste estudo $(5,73$ para os jovens e 2,66 para os DVs) percebe-se nitidamente melhor resultado dos DVs, reforçando o bom nível proprioceptivo dos sujeitos deste grupo, através das sensações geradas pelos mecanoreceptores localizados na pele, músculos, ossos e tendões.

A importância de treinamento físico que desenvolva a propriocepção é observada no estudo sobre coordenação motora fina e propriocepção em idosas praticantes de hidroginástica entre 59 e 84 anos, realizado por Antes, Katzer e Corazza ${ }^{30}$, que constataram que o grupo com idade mais avançada teve desempenho inferior nos testes, demonstrando perdas motoras com o avanço da idade.

As limitações encontradas nesse estudo estão relacionadas ao pequeno número de sujeitos, dificultando a generalização dos resultados e à pequena variação de exercícios específicos para a propriocepção de membros superiores. Sugere-se, desta forma, um olhar atento a estes elementos em futuras pesquisas com indivíduos com DV.

\section{CONCLUSÕES}

Ao analisar os efeitos de um programa de treinamento funcional na propriocepção e no equilíbrio de pessoas com deficiência visual, podese constatar que houve melhora no equilíbrio e na propriocepção de membros inferiores dos sujeitos, o que confirma a eficácia do programa de treinamento funcional com este pequeno grupo.

Nesse contexto, o treinamento funcional é uma possibilidade de propiciar a inclusão da pessoa com DV às práticas corporais, contribuindo para a melhoria de sua qualidade de vida, sociabilidade, saúde, liberdade e autonomia.

Todos os autores declararam não haver qualquer potencial conflito de interesses referente a este artigo.

CONTRIBUIÇÕES DOS AUTORES: Cada autor contribuiu individual e significativamente para o desenvolvimento do manuscrito. STC (0000-0002-2684-2412)* realizou a revisão crítica do artigo, aprovação final da versão do manuscrito. RCL (0000-0003-2723-3130)* análise e interpretação dos dados, desenho e aprovação final. MLSF (00000003-3165-1183)* redação e interpretação dos dados. DLS (0000-0002-1782-1337)* revisão crítica do artigo e desenho do trabalho. RRM (0000-0001-8001-6333)* aplicação da intervenção com treinamento funcional, desenho do estudo e pesquisa bibliográfica. TLM (0000-0002-1953-7794)* revisão crítica do artigo, interpretação dos dados e pesquisa bibliográfica. ACP (0000-0001-8225-3279)* coleta de dados, tratamento dos dados da plataforma de força e pesquisa bibliográfica. SPM (0000-0002-1019-0237)* coleta e tratamento dos dados da propriocepção e pesquisa bibliográfica. *ORCID (Open Researcher and Contributor ID).

\section{REFERÊNCIAS}

1. World Health Organization. Visual impairment and blindness.2014. [acesso em 2016 fev. 2];FactSheet №282. Disponível em: http://www.who.int/mediacentre/factsheets/fs282/en/

2. Portal Brasil. Portal do Governo Federal. Dia Mundial da Visão alerta para a prevenção da cegueira no País. 2012. [acesso em 2016 fev. 2];Disponível em: http://www.brasil.gov.br/saude/2012/10/ dia-mundial-da-visao-alerta-para-a-prevencao-da-cegueira-no-pais

3. Scherer RL, Rodrigues LA, Fernandes LL. Contribuição do goalball para orientação e mobilidade sob a percepção dos atletas de goalball. Pensar a prática. 2011;14(3):115.

4. Lopes MCB, Kitadai SPS, Okai LA. Avaliação e tratamento fisioterapêutico das alterações motoras presentes em crianças deficientes visuais. RevBrasOftal.2004;63(3):155-61.

5. Alves MLT, Duarte E. A inclusão do deficiente visual nas aulas de educação física escolar: impedimentos e oportunidades. Acta Sci Human Soc Sci. 2005;27(2):231-7.

6. Craft DH. Sensory imparmeints. In:Joseph P. Winnick, editor.Adapted physical education and sports. Champaign: Human Kinectics Books; 1990.
7. Conde AJM. A pessoa portadora de deficiência visual, seu corpo, seu movimento e seu mundo. In: Pedrinelli VJ et al., editor. Educação física e desporto para pessoas portadoras de deficiência. Brasilia: MEC-Sedes, Sesi-DN;1994.

8. Almeida JJG. Estratégias para aprendizagem esportiva: uma abordagem pedagógica da atividade motora para cegos e deficientes visuais (Tese de Doutorado). Campinas: Faculdade de Educação Física, Universidade Estadual de Campinas;1995.

9. Schmidt RA, Wrisberg CA. Aprendizagem e Performance Motora: uma abordagem da aprendizagem baseada na situação. Trad. Ricardo Demétrio de Souza Peterson. 4a ed. Porto Alegre: Artmed;2010.

10. Horak FB, Macpherson JM. Postural orientation and equilibrium. In: Rowell LB, Shepherd JT, editors Handbook of physiology: a critical, comprehensive presentation of physiological knowledge and concepts. New York:Oxford American Physiological Society;1996.

11. Fatoye F, Palmer S, Macmillan F, Rowe P, vander Linden M. Proprioception and muscle torque deficits in children with hypermobility syndrome. Rheumatology. 2009;48(2):152-7. 
12. Lee DN. Visuo-motor coordination in space-time. In StelmachGE, Requin J, editors. Tutorials in Motor Behavior(pp. 281-293). Amsterdam: North- Holland;1990.

13. Ganança FF, Castro ASO, Branco FC, Natour J. Interferência da tontura na qualidade de vida de pacientes com síndrome vestibular periférica. Ver Bras Otorrinolaringol. 2004;70(1):94-101.

14. Soares FA, Silva TR, Gomes DP, Pereira ET. A contribuição da estimulação psicomotora para o processo de independência do deficiente visual. Motri. 2012;8(4):16-25.

15. Bastos LSC, Gaio RC. Técnicas de orientação e mobilidade para pessoas cegas: reflexões na perspectiva da educação física. Movimento \& Percepção. 2010;11(16):120-47.

16. Seabra Júnior MO, Zengo LM, Fortaleza ACS, Silva CB, Freitas IF, Zengo LC. Análise do equilíbrio na marcha de pessoas cegas. Rev Eletr Gestão \& Saúde. 2013;1(ESP):624-33.

17. Teotonio JJSO, Blumer LM, Santos MS, Carvalho TB, Viana HB. Treinamento funcional: benefícios, métodos e adaptações. EFDeportes. 2013;178.

18. Yin RK. Estudo de caso: planejamento e métodos.4a ed. Porto Alegre: Bookman;2010.

19. Suominen V, Salenius J, Sainio P, Reunanen A, Rantanen T. Peripheral arterial disease, diabetes and postural balance among elderly Finns: a population-based study. Aging Clin Exp Research. 2008;20(6):540-6

20. Cunha AG, Nunes MP, Ramos RT, Carvalo-Pinto RM, Boffino CC, Martins FC, et al. Balance disturbances in asthmatics patients. JAsthma. 2013;50(3):282-6.

21. Paixão JS. Efeitos do plano motor na aquisição, retenção e transferência de uma destreza fechada
[Dissertação de Mestrado], Santa Maria: Universidade Federal de Santa Maria;1981.

22. Deshpande N, Connelly DM, Culham EG, Costigan PA. Reliability and validity of ankle proprioceptive measures. Arch Phys Med Rehabil.2003;84(6):883-9.

23. Seabra Jr MO, Manzini EJ. Recursos e estratégias para o ensino do aluno com deficiência visual na atividade física adaptada. Marília: ABPEE;2008.

24. Silva CAC, Ribeiro GM, Rabelo RJ. A influência da dança no equilíbrio corporal de deficientes visuais. Movimentum: Rev Dig Educ Fís.2008;3(1):1-8.

25. Souza CM, Oliveira Filho CW, Ferreira ACGO, Almeida JJG. Ginástica artística para crianças deficientes visuais: relato de experiência. EFDeportes.2006;94.

26. Silva MCR, Corazza ST, Katzer JI, Mota CB, Soares JC. Equilíbrio corporal em crianças e adolescentes asmáticos e não asmáticos. Motriz. 2013;19(2):480-6.

27. Antes DL, Wiest MJ, Mota CB, Corazza ST. Análise de estabilidade postural e propriocepção de idosas fisicamente ativas. Fisio Mov. 2014;27(4):531-9.

28. Oliveira DN, Barreto RR. Avaliação do equilíbrio estático em deficientes visuais adquiridos. Rev Neurociências. 2005:13(3):122-7.

29. Antes DL, Contreira AR, Katzer Jl, Corazza ST. Propriocepção de joelho em jovens e idosas praticantes de exercícios físicos. Fisioter Pesq. 2009;16(4):306-10.

30. Antes DL, Katzer Jl, Corazza ST. Coordenação motora fina e propriocepção de idosas praticantes de hidroginástica. Rev Bras Cien Envelhec Hum. 2008;5(2):24-32.

\section{ERRATA}

No artigo intitulado "BENEFÍCIOS DO TREINAMENTO FUNCIONAL PARA O EQUILÍBRIO E PROPRIOCEPÇÃO DE DEFICIENTES VISUAIS" de autoria de Sara Teresinha Corazza; Rafael Cunha Laux, Miriam Léa Struass Foesch; Daniela Lopes dos Santos; Rafaella Righes Machado; Thuane Lopes Macedo; Ana Carla Piovesan; Stela Paula Mezzomo, publicado na Revista Brasileira de Medicina do Esporte (RBME) vol.22 n 6, 2016, página 471, DOl: http:// dx.doi.org/10.1590/1517-869220162206164644, por solicitação dos autores.

Onde se lê: Ana Carla Piovesam

Leia-se: Ana Carla Piovesan 